\title{
Helping people with schizophrenia to quit smoking
}

\author{
Toshi A Furukawa
}

Departments of Health Promotion and Human Behavior and of Clinical Epidemiology, Kyoto University Graduate School of Medicine/

School of Public Health, Kyoto, Japan; furukawa@kuhp.kyoto-u.ac.jp

\section{CLINICAL CASE}

\section{Patient}

anhedonia) and some residual positive symptoms (vague and distant auditory hallucinations). He does not suffer from substance-related disorders other than tobacco use disorder.

\section{FORMULATE YOUR CLINICAL QUESTION}

Patients: Patients with schizophrenia who smoke

Intervention: Pharmacological intervention

Comparison: Placebo

Outcomes: Continued abstinence from smoking.

\section{LITERATURE SEARCH}

Ideally, clinical decisions should be based on the totality of evidence and not on individual trials. You therefore start looking for a study that tries to look at the total body of evidence, a systematic review.

Being uncertain if treatments shown to help people to quit smoking in general may not have the same effectiveness and may have different adverse effects for people with schizophrenia, you decide to look for a systematic review that specifically looked at interventions for smoking cessation among people with schizophrenia. Visiting PubMed and searching its MeSH database, you notice that "smoking cessation" is a MeSH term and you enter "smoking cessation"[mesh] and "schizophrenia" [mesh] in Clinical Queries. The second under the heading "Systematic Reviews" is a Cochrane review on this very topic. ${ }^{1}$ 
In order to appraise the totality of evidence, we will follow the new Users' Guide recently published in JAMA. ${ }^{2}$

First judgement: evaluate the credibility of the methods of systematic review

Did the review explicitly address a sensible clinical question?

YES. The clinical question of this review may be summarised as:

P: patients with schizophrenia or schizoaffective disorder

I: pharmacological or non-pharmacological intervention aiming

at smoking cessation or reduction

C: another intervention, placebo or usual care

0 : smoking cessation.

Our original clinical question PICO is not exactly the same as this review's PICO, but we expect that the latter covers the former and would like to read on.

Was the search for relevant studies exhaustive?

YES. The review authors searched the Cochrane Tobacco Addiction Group Specialized Register and several electronic databases, in addition to clinical trials registers and manual searching. The search terms in the appendix look comprehensive.

\section{Were selection and assessments of studies reproducible?}

YES/NO. The selection and assessments were performed by two or more independent authors. However, the review authors do not report their reproducibility (inter-rater agreement).

\section{Did the review present results that are ready for clinical} application?

YES. The results were reported separately for individual interventions; only results for bupropion were summarisable in a clinically meaningful way. They are provided in forest plots as RRs and in the summary of the findings table as Numbers Needed to Treat (NNTs).

\section{Did the review address confidence in estimates of effect?}

YES. The review authors provided enough information for the readers to judge confidence in the study results, such as risks of bias for individual trials and heterogeneity among the included trials.
Second judgement: rate the confidence in the effect estimates

How serious is the risk of bias in the body of evidence? NOT SERIOUS. Cochrane reviews assess and present risks of bias of each included study in the domains of random sequence generation, allocation concealment, blinding of participants and personnel, blinding of outcome assessment, incomplete outcome data, and selective reporting. The five studies included for the comparison of bupropion versus placebo were judged to be mostly at either a low or unclear risk of bias.

Are the results consistent across studies?

YES. The forest plot for the comparison of bupropion versus placebo presents little variability from study to study, and the $\mathrm{I}^{2}$ statistic was estimated to be $0 \%$.

How precise are the results?

NOT VERY PRECISE. The pooled RR of bupropion over placebo (with or without a nicotine patch) for abstinence at 6-month follow-up was 2.78 (1.02 to 7.58). While this estimate is 'statistically significant,' the lower end of its $95 \% \mathrm{Cl}$ is close to unity (no difference between the two arms) and would indicate no clinically meaningful benefit of bupropion over placebo. The estimate is therefore not precise.

\section{Do the results directly apply to my patient?}

YES. The average age of the included patients was in the 40 s; they had been diagnosed as suffering from schizophrenia or schizoaffective disorder according to Diagnostic and Statistical Manual Fourth Edition (DSM-IV) and had expressed interest in quitting. There appears to be no strong reason to suspect that the results of the study would not apply to the patient in the clinical scenario.

\section{Is there concern about reporting bias?}

PROBABLY NO. The number of the included studies ( 5 out of 7 relevant trials) is too small to statistically examine the possibility of publication bias. The literature search appears to be very comprehensive, but in the current practice of the medical literature there always remains some doubt as to the completeness of the included evidence.

\section{WHAT WILL YOU DO WITH YOUR PATIENT?}

While the point estimate of bupropion over placebo in bringing about abstinence from smoking at 6-month follow-up appears to be impressive $(R R=2.78)$, the lower limit of its $C I$ is only marginally above 1.0. Moreover, given the very low success rate in the placebo group (approximately $5 \%$ ), this RR would translate into NNT of 12 (3-1000), using the following formulae.

$\mathrm{NNT}=1 /$ Risk difference

Risk difference $=$ Control event rate $\times \mathrm{RR}-$ Control event rate where Control event rate $=5 \%, \mathrm{RR}=2.78$ (and its upper or lower confidence limits).

On the other hand, this review noted that there were no significant differences in schizophrenic or depressive symptoms between bupropion and placebo. In the included trials, as reported in the literature for bupropion in general, the drug was associated with insomnia, restlessness and dry mouth among others. You conclude that there is moderate quality evidence that bupropion would bring an important yet small benefit for people with schizophrenia who smoke but who would like to quit smoking. After some discussion with you, your patient decided to give bupropion a try.

Competing interests TAF has received lecture fees from Eli Lilly, Meiji, Mochida, MSD, Pfizer and Tanabe-Mitsubishi, and consultancy fees from Sekisui and Takeda Science Foundation. He is a diplomate of the Academy of Cognitive Therapy. He has received royalties from Igaku-Shoin, Seiwa-Shoten and Nihon Bunka Kagaku-sha. The Japanese Ministry of Education, Science, and Technology, the Japanese Ministry of Health, Labour and Welfare and the Japan Foundation for Neuroscience and Mental Health have funded his research projects.

doi:10.1136/eb-2014-102022

\section{REFERENCES}

1. Tsoi DT, Porwal M, Webster AC. Interventions for smoking cessation and reduction in individuals with schizophrenia. Cochrane Database Syst Rev 2013;2:CD007253.

2. Murad MH, Montori VM, loannidis JP, et al. How to read a systematic review and meta-analysis and apply the results to patient care: users' guides to the medical literature. JAMA 2014;312:171-9. 